\title{
An Experimental Investigation of Internal Tide Generation by Two-Dimensional Topography
}

\author{
Thomas Peacock and Paula Echeverri \\ Massachusetts Institute of Technology, Cambridge, Massachusetts \\ NeIL J. BALMFORTH \\ University of British Columbia, Vancouver, British Columbia, Canada
}

(Manuscript received 11 December 2006, in final form 23 February 2007)

\begin{abstract}
Experimental results of internal tide generation by two-dimensional topography are presented. The synthetic Schlieren technique is used to study the wave fields generated by a Gaussian bump and a knife edge. The data compare well to theoretical predictions, supporting the use of these models to predict tidal conversion rates. In the experiments, viscosity plays an important role in smoothing the wave fields, which heals the singularities that can appear in inviscid theory and suppresses secondary instabilities of the experimental wave field.
\end{abstract}

\section{Introduction}

Two-dimensional (2D) ridges are considered to be the strongest generators of baroclinic (internal) tides because barotropic tidal currents cannot easily flow around and must therefore flow over them (Holloway and Merrifield 1999). The internal tides radiated by such ridges are characterized by well-defined wave beams at the tidal frequency (Nash et al. 2006), and their eventual degradation is believed to play an important role in ocean mixing (Garrett and Kunze 2007). A detailed understanding of the nature of the internal wave fields generated by ridgelike topography is therefore an important aspect of tidal conversion.

In a deep ocean there are four nondimensional parameters that control 2D internal tide generation (Garrett and Kunze 2007). The first two parameters relate the characteristic frequencies of the system: $\omega / f$ and $\omega / N$, where $\omega$ is the tidal frequency, $f$ is the Coriolis frequency, and $N$ is the Brunt-Väisälä frequency. These two parameters govern the angle $\theta$ of propagation of internal gravity waves with respect to the horizontal via the dispersion relation,

Corresponding author address: Thomas Peacock, 1-310D, Mechanical Engineering, MIT, Cambridge, MA 02139.

E-mail: tomp@mit.edu

$$
\tan \theta=\sqrt{\frac{\omega^{2}-f^{2}}{N^{2}-\omega^{2}}},
$$

such that waves only propagate if $f<\omega<N$. The other two parameters relate spatial scales. The "criticality parameter," $\varepsilon=h_{\max }^{\prime} / \alpha$, is the ratio of the maximum slope, $h_{\max }^{\prime}$, of the ocean topography, $h(x)$, to the slope of the radiated wave beam, $\alpha=\tan \theta$. The "excursion parameter," $u_{0} /(\omega l)$, is the ratio of the tidal displacement $a=u_{0} / \omega, u_{0}$ being the maximum tidal velocity, to the horizontal scale of the topography $l$.

When the excursion parameter is small, the governing fluid equations become linearized, and the conversion problem is dramatically simplified. As this regime is relevant to most ocean ridges, many theoretical results focus on this particular limit of the problem and consider either "subcritical" $(\varepsilon<1)$ or "supercritical" $(\varepsilon>1)$ topography. Subcritical topography has slopes that are everywhere less than the slope of the internal wave beams, whereas the slope of supercritical topography somewhere exceeds that inclination. Subcritical topography generates smooth wave profiles (Balmforth et al. 2002). Tidal conversion by supercritical topography, on the other hand, is characterized by wave beams that become singular along rays emanating from the positions on the topography where the angle

DOI: $10.1175 / 2007 J P O 3738.1$ 


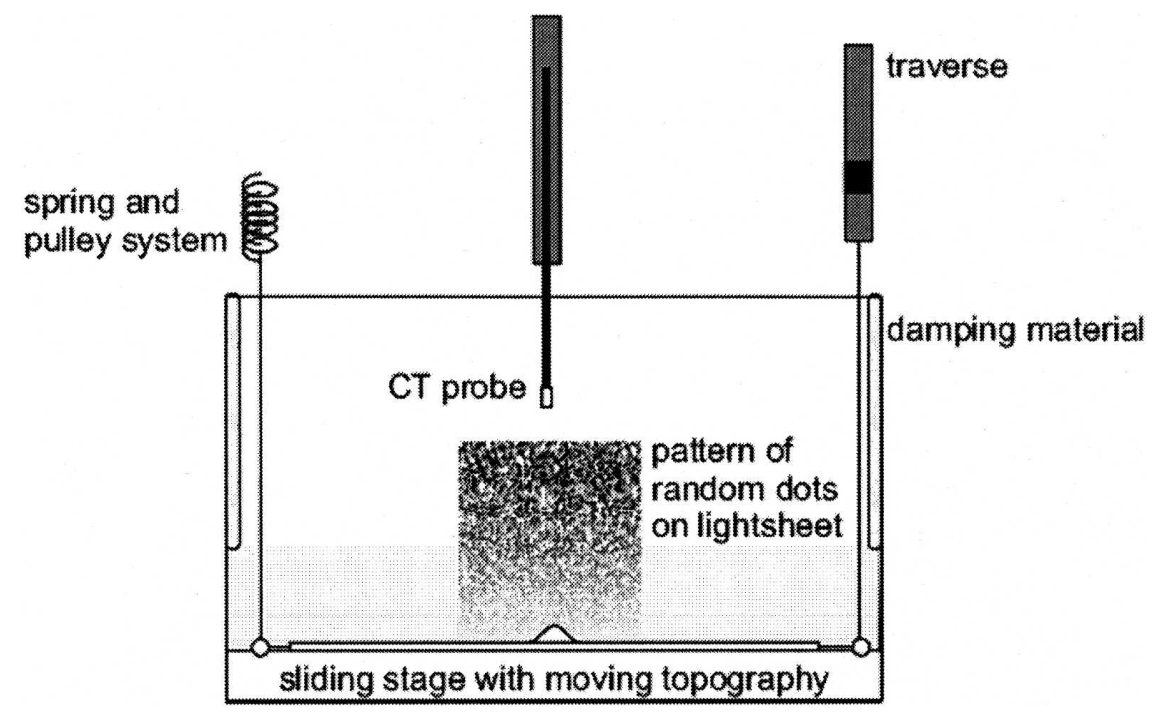

FIG. 1. Schematic illustration of the experimental apparatus. A density stratification was established in the experimental tank, and an internal wave field was generated by topography mounted on a sliding stage at the base of the tank.

of the slope equals $\theta$ (Baines 1982; St. Laurent et al. 2003; Llewellyn Smith and Young 2003; Petrelis et al. 2006).

Our goal in the current work is to present a laboratory exploration that complements these theoretical studies. We consider two topographic configurations, designed to test separately the sub- and supercritical theories. For subcritical topography, we use a Gaussian-shaped bump; for supercritical topography, we use a knife edge. ${ }^{1}$ The experiments utilize the synthetic Schlieren technique (Sutherland et al. 1999); further details are presented in section 2. Section 3 summarizes the theoretical formulation of the problem. Sections 4 and 5 compare theory and experiment for the Gaussian and knife-edge topographies, and in section 6 we draw our conclusions.

\section{Experimental arrangement}

The experiments were performed in a 1.32 -m-wide, 0.2 -m-thick, and 0.66-m-high Plexiglas tank, as illustrated in Fig. 1. An ultrahigh-molecular-weight (UHMW) polyethylene sliding stage was placed on the base of the tank and connected via a spring-and-pulley

\footnotetext{
${ }^{1}$ After submitting this article for publication, we became aware of two related, more recent studies: Gostiaux and Dauxois (2007) perform an experimental exploration of wave generation at a continental shelf. Zhang et al. (2007) report experiments with an oscillating cylinder [in the fashion of Sutherland et al. (1999)]; by oscillating this object horizontally, they offer a visualization of wave generation by a supercritical ocean ridge.
}

system to a motion-controlled traverse. The sliding stage and the traverse were levelled to within $0.1 \pm$ $0.02^{\circ}$ of the horizontal using a spirit level. Topographic features were mounted in the central section of the sliding stage, which was subsequently oscillated from side to side. The topographic features used were a Gaussian bump of height $h=14.7 \pm 0.2 \mathrm{~mm}$ and standard deviation $\sigma=20 \pm 0.2 \mathrm{~mm}$, cut out of closed-cell foam, and a knife edge of height $h=16.5 \pm 0.2 \mathrm{~mm}$ and width $l=1.28 \pm 0.02 \mathrm{~mm}$, machined out of stainless steel. For each experiment, the millimeter amplitude oscillation $a$ of the topography was tracked using a video camera. Blocksom-filter matting was placed at the end walls of the tank and along the surface to provide highly effective damping of internal gravity waves.

A linear stratification was established using the Oster double-bucket technique (Oster 1965) and was not noticeably affected by the presence of the topography, which was much smaller than the size of the wave tank. The Brunt-Väisälä frequency $N$ was determined using both a Precision Measurement Engineering (PME) conductivity probe and by measuring the wave beam angles, $\theta$, for a given forcing frequency. Typical values of $N$ and $\omega$ used were $1.25 \mathrm{rad} \mathrm{s}^{-1}$ and between 0.60 and $1.0 \pm 0.01 \mathrm{rad} \mathrm{s}^{-1}$, respectively. Two independent techniques, a cone-and-plate rheometer and a glass capillary kinematic viscometer, were used to measure the kinematic viscosity of the saltwater under operating conditions, which was found to be $\nu=1.10 \pm 0.02 \times$ $10^{-6} \mathrm{~m}^{2} \mathrm{~s}^{-1}$. The Reynolds number for these experiments, based on the topographic height, was $\mathrm{Re}=$ $a \omega h / \nu \sim O\left(10^{1}\right)$. 
Quantitative data were obtained using the synthetic Schlieren technique (Sutherland et al. 1999). A random pattern of $0.5-\mathrm{mm}$-diameter dots was printed on a transparency backlit by a $0.30-\mathrm{m}$ by $0.25-\mathrm{m}$ Perfalite electroluminescent sheet and positioned a distance $B=$ $0.955 \mathrm{~m}$ behind the tank. Apparent distortions of the pattern, caused by the wave field, were captured using a JAI CV-M4 + CL CCD camera with a resolution of 1268 by 1024 pixels. The camera was placed a distance $L=3.50 \mathrm{~m}$ in front of the tank so that area of the pattern imaged subtended a half-angle of less than $2^{\circ}$. Movies were processed using DigiFlow software (see http://www.damtp.cam.ac.uk/lab/digiflow/) to determine the perturbations to the stratification $\Delta N^{2}=-(g /$ o) $d \rho / d z$.

Noise levels were reduced to less than $2 \%$ of the characteristic experimental values of $\Delta N^{2}$ by taking the following precautions: ambient thermal fluctuations were minimized by turning off the air conditioning in the laboratory during an experiment and placing a 1.5$\mathrm{m}$-long thermal isolation tunnel in front of the camera; intensity fluctuations of rms amplitude 3/256 in the light sheet were countered by achieving a high contrast, in excess of 110/256 between light and dark regions in the random pattern; and $\Delta N^{2}$ was averaged over the data from the same phase in four consecutive periods (this final procedure also confirmed that the wave field had reached steady state). To check the performance of our synthetic Schlieren arrangement we successfully repeated the oscillating cylinder experiments of Sutherland et al. (1999) and Dalziel et al. (2000).

\section{Governing equations}

In the limit of a small excursion parameter, and assuming the Boussinesq approximation, the governing linearized equations for wave motion in a $2 \mathrm{D}$, stratified fluid can be written in the form:

$$
\begin{gathered}
u_{t}+p_{x}=\nu \nabla^{2} u, \\
w_{t}-b+p_{z}=\nu \nabla^{2} w, \\
b_{t}+N^{2} w=0, \quad \text { and } \\
u_{x}+w_{z}=0,
\end{gathered}
$$

where $(u, w)$ is the velocity field, $p$ and $b$ are the associated variations in pressure (divided by a reference density) and buoyancy, $\nu$ is the viscosity, and $x$ and $z$ are the horizontal and vertical coordinates, respectively. Here we have neglected background rotation, which was not present in the experiments, and retained viscosity. By defining a streamfunction $\psi(x, z)$, such that $\mathbf{u}(x, z, t)=\operatorname{Re}\left\{\left[-\psi_{z}(x, z), \psi_{x}(x, z)\right] e^{-i \omega t}\right\}$, Eqs. (2) $-(5)$ can be reduced to the internal wave equation,

$$
\omega^{2} \nabla^{2} \psi(x, z)-N^{2} \psi_{x x}(x, z)=i \omega \nu \nabla^{4} \psi(x, z) .
$$

Since the amplitude of the topography $(\sim 15 \mathrm{~mm})$ was small relative to the depth of the tank $(\sim 0.6 \mathrm{~m})$ in the experiments, we assume a semi-infinite domain, $h(x)<z<\infty$. This demands the imposition of a radiation condition as $z \rightarrow \infty$ for the inviscid problem $(\nu=0)$, or that amplitudes decay if $\nu \neq 0$. We must also impose boundary conditions on the topography at $z=h(x)$. Inviscid theory requires that flow follow the topographic slope:

$$
\psi[x, h(x)]=u_{0} h(x) .
$$

In a full viscous model, the no-slip condition is more appropriate.

\section{The Gaussian bump}

\section{a. Analytical solution}

Balmforth et al. (2002) solve the inviscid version of (6) by adopting periodic boundary conditions in $x$ and introducing the Fourier series solution

$$
\psi(x, z)=\sum_{n=1}^{\infty} a_{n} e^{m z} \cos (n \kappa x),
$$

where $m=-i n \mu \kappa, \kappa=2 \pi / L, \mu=\sqrt{\left(N^{2} / \omega^{2}\right)-1}$, and $L$ is the length of the periodic domain. The coefficients $a_{n}$ can be found by substituting the series into Eq. (7) and solving the resulting integral equation by Fourier projection and matrix methods (see Balmforth et al. 2002). The solution assumes a priori that there are only upgoing waves and cannot, therefore, deal with supercritical topography. For the topography, one can take

$$
h(x)=h_{0} e^{-\gamma(1-\cos \kappa x)},
$$

which, for large values of the dimensionless parameter $\gamma$, turns into a periodic array of nearly Gaussian bumps with height $h_{0}$ and standard deviation $\sigma=\kappa / \sqrt{\gamma}$.

To incorporate the effects of weak viscosity, we follow the lead of Hurley and Keady (1997) and assume that there is a passive Stokes boundary layer of thickness $O(\sqrt{\nu / \omega})$ above the topography in which the solution varies sharply to satisfy the no-slip condition but, otherwise, does not affect the solution. The far-field inviscid solution is then obtained by replacing the original exponent $m$ in (8) with

$$
m \approx-i n \kappa \mu\left(1+\frac{i \nu n^{2} \kappa^{2} N^{4}}{2 \mu^{2} \omega^{5}}\right) .
$$


This exponent is the relevant approximate root to the dispersion relation that follows from Eq. (6). ${ }^{2}$

Equation (8) incorporating the viscous correction in Eq. (10) applies to periodic topography. However, when the Gaussians are widely separated it is more appropriate to split Eq. (8) into two components that represent the left- and right-going wave beams (achieved by writing the cosine in terms of its constituent exponentials) and to apply a viscous correction to each separately. The right-going wave beam, for example, must then be corrected according to the distance traveled along the beam, rather than in $z$ alone. We thus write

$$
\psi(x, z)=\sum_{n=1}^{\infty} a_{n} e^{\left[i n \kappa(x-\mu z) \frac{v n^{3} \kappa^{3} N^{4}(x+\mu z)}{2 \mu^{2} \omega^{5}}\right]}
$$

and from this compute $\Delta N^{2}=\partial b / \partial z$ for comparison with experiment.

\section{b. Experimental results}

The Gaussian topography described in section 2 was cut to have a maximum slope angle of $24^{\circ}$. Here, we present results for two experiments performed using this topography for subcritical and near-critical values of $\varepsilon$.

In the first experiment, we set $\varepsilon=0.30 \pm 0.01$ by virtue of $N=1.23 \pm 0.01 \mathrm{rad} \mathrm{s}^{-1}$ and $\omega=0.98 \mathrm{rad} \mathrm{s}^{-1}$, resulting in a wave beam angle of $\theta=53.3 \pm 0.2^{\circ}$. The horizontal scale of the topography was $l=4 \sigma=80.0$ $\mathrm{mm}$ and the forcing amplitude was $a=2.79 \pm 0.02 \mathrm{~mm}$, giving a small excursion parameter $2 a / l \approx 0.06$. Figure 2 presents a contour plot of $\Delta N^{2}$ for a radiated wave beam at an instant when the topography was at the leftmost point of its oscillation. Henceforth, we define the phase of the oscillation $\phi$ to be zero at the instant when the topography is at the midpoint of its oscillation moving right to left, which corresponds to maximum tidal velocity in the positive $x$ direction in the frame of reference moving with the topography (this being the usual frame of reference for analytical models). The corresponding phase of oscillation for Fig. 2 is therefore $\phi=\pi / 2$.

As a quantitative comparison between experiment and theory, we take cross-sectional profiles at the locations indicated by lines 1 and 2 in Fig. 2, whose centers are a distance $4 \sigma$ and $12 \sigma$ from the origin of the Gaussian topography, respectively. The results are presented

\footnotetext{
${ }^{2}$ Of the four roots to this dispersion relation, one grows as $z \rightarrow \infty$, two are associated with the Stokes layer, and the remaining root is the perturbed inviscid solution, $m=-i n \kappa \mu$.
}

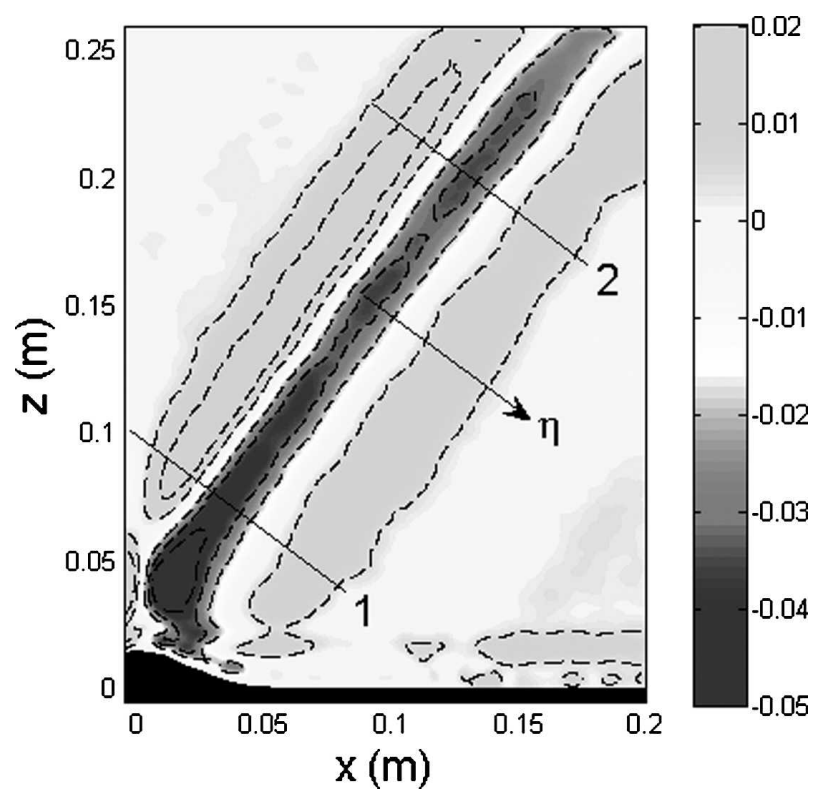

FIG. 2. Experimental $\Delta N^{2}$ field for the Gaussian bump with $\varepsilon=$ $0.30, \phi=\pi / 2$, and $\theta=53.3^{\circ}$. Lines 1 and 2 indicate the location of cross-sectional profiles. Contours are plotted at intervals of 0.0125 between -0.045 and $0.0175 ; \eta$ is a cross-beam coordinate used in Fig. 3.

in Fig. 3. Profiles are presented for two different phases, $\phi=0$ and $\pi / 2$, after transient waves from the first few oscillations have disappeared and the wave field is periodic. In agreement with theory, the profiles at $\phi=\pi$ and $3 \pi / 2$ were the inverse of those taken at $\phi=0$ and $\pi / 2$, respectively.

In general, the viscous theory compares well with the experimental results and is superior to the inviscid theory, which is also shown in Fig. $3 .^{3}$ The viscous theory overpredicts the perturbation amplitude in the sharp peaks and troughs, a trend that has also been reported for related studies of the wave fields generated by oscillating circular and elliptical cylinders (Dalziel et al. 2000; Sutherland and Linden 2002).

For the second, near-critical experiment, the stratification was $N=1.24 \pm 0.01 \mathrm{rad} \mathrm{s}^{-1}$, and the forcing frequency was $\omega=0.59 \mathrm{rad} \mathrm{s}^{-1}$. The resulting wave beams had an angle of $\theta=28.3 \pm 0.2^{\circ}$, giving $\varepsilon=$ $0.83 \pm 0.01$, and the amplitude of oscillation and tidal excursion parameter were the same as in the first experiment. Significantly, this value of $\varepsilon$ is the lowest for which inviscid theory predicts gravitationally unstable

\footnotetext{
${ }^{3}$ The "inviscid theory" here is actually a weakly viscous solution with $\nu=10^{-8} \mathrm{~m}^{2} \mathrm{~s}^{-1}$, which we chose to ensure nonsingular beams in the knife-edge solution. For consistency, we have also used this value for the Gaussian bump and henceforth refer to this as the inviscid solution.
} 

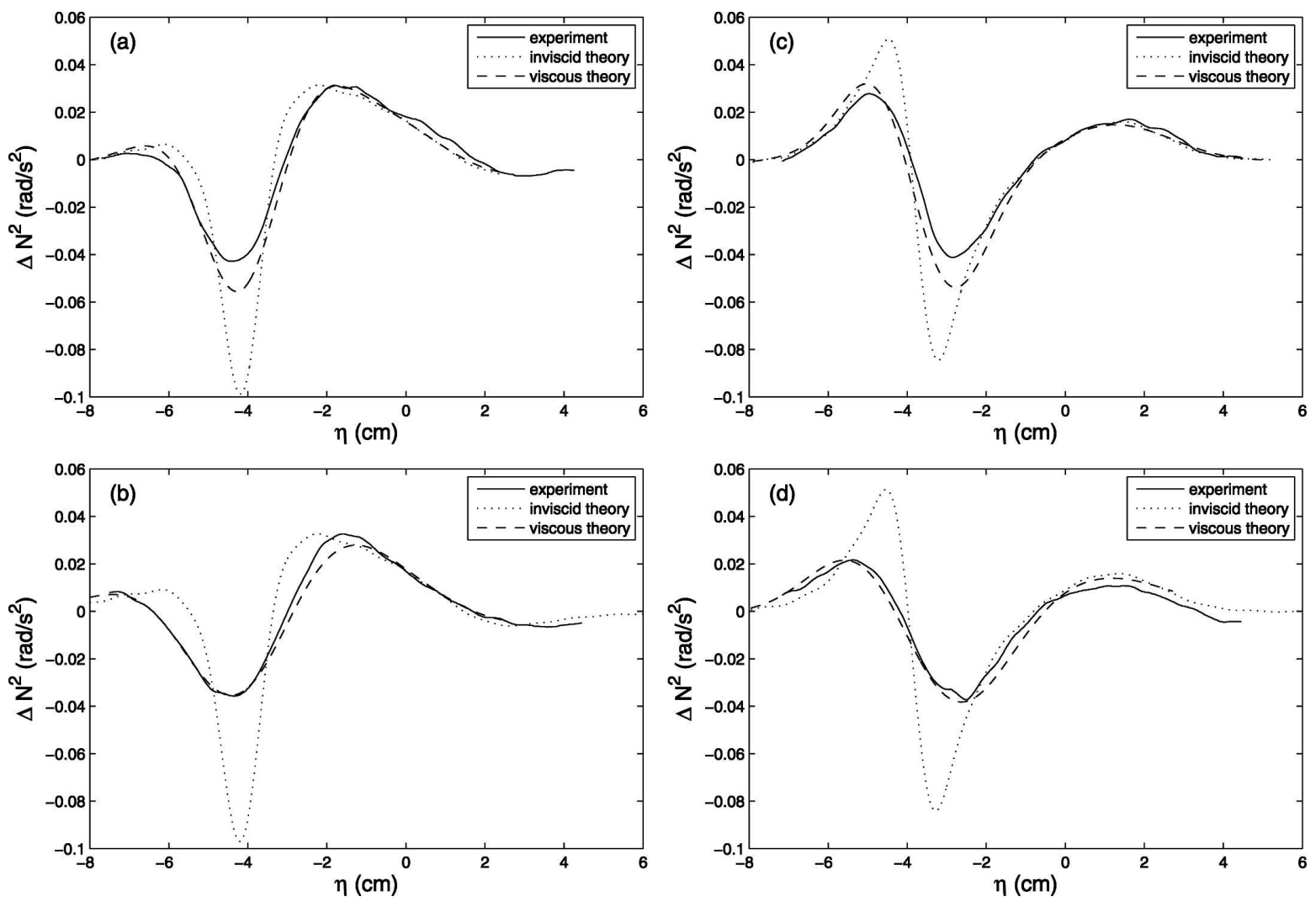

FIG. 3. Profiles of $\Delta N^{2}$ for cross sections 1 and 2 in Fig. 2 with $\phi=0$ and $\pi / 2 ; \eta$ is the cross-beam coordinate that increases with increasing $x$.

wave beams. Figure 4 presents a contour plot of the $\Delta N^{2}$ field and the locations of cross sections 1 and 2 , along which theory and experiment are compared for $\phi=0$ and $\pi / 2$ in Fig. 5. Again we see that there is good agreement between experiment and theory, provided viscous effects are included. As one might expect, the wave field is noticeably stronger than that for the more subcritical arrangement, although there is no gravitational instability.

\section{The knife edge}

\section{a. Analytical solution}

Predictions of the wave beams generated by knifeedge topography can be extracted from the solution for an oscillating ellipse by Hurley and Keady (1997). By suitably orienting the ellipse and collapsing one of the semi-axes to zero, we arrive at the streamfunction for the knife edge:

$$
\psi(x, z)=-\frac{i u_{0} h_{0}}{2} \times \int_{0}^{\infty} \frac{J_{1}(k)}{k} \exp \left[-\left(\frac{k \alpha}{h_{0}}\right)^{3} \frac{\nu(x+\alpha z)\left(1+1 / \alpha^{2}\right)}{2 \omega}+i \frac{k \alpha}{h_{0}}\left(x-\frac{z}{\alpha}\right)\right] d k,
$$

which holds in the upper right-hand quadrant $(x, z>0)$ in regions where there are only upwardly propagating waves. To find $\Delta N^{2}$, the integrand of Eq. (12) is multiplied by the prefactor

$$
\begin{aligned}
N^{2}\left(\frac{k \alpha}{h_{0}}\right)^{2} & {\left[-\nu\left(\frac{k \alpha}{h_{0}}\right)^{2} \frac{1+1 / \alpha^{2}}{2 \omega}+i\right] } \\
\times & {\left[-\nu \alpha\left(\frac{k \alpha}{h_{0}}\right)^{2} \frac{1+1 / \alpha^{2}}{2 \omega}-\frac{i}{\alpha}\right] }
\end{aligned}
$$

and the integral evaluated numerically.

\section{b. Experimental results}

The knife edge was oscillated side to side with amplitude $a=0.88 \pm 0.02 \mathrm{~mm}$ and frequency $\omega=0.836$ $\mathrm{rad} \mathrm{s}^{-1}$ in a stratification of $N=1.18 \pm 0.01 \mathrm{rad} \mathrm{s}^{-1}$, generating wave beams angled at $\theta=44.9 \pm 0.3^{\circ}$ to the horizontal. Figure 6 shows the experimental $\Delta N^{2}$ field for the knife edge (at $\phi=0$ ), for which there is a more noticeable spreading of the beam than for the Gaussian bumps previously studied. Figure 7 presents a comparison between experiment and theory along cross sec- 


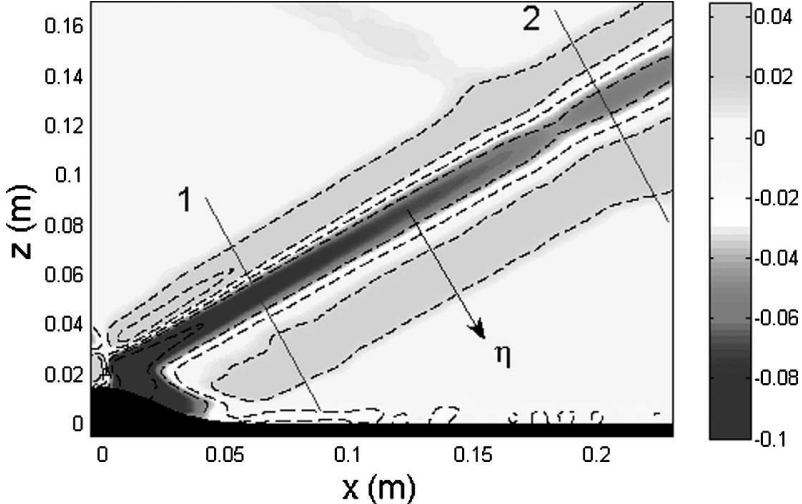

FIG. 4. As in Fig. 2, but with $\varepsilon=0.83, \phi=\pi / 2$, and $\theta=28.3^{\circ}$. Contours are plotted at intervals of 0.03 between -0.105 and 0.045 . The weak beam (upper middle), oriented in the opposite direction to the main beam, originated from the edge of the oscillating stage.

tions through the beams at locations 1 and 2, whose centers are $3 h_{0}$ and $10 h_{0}$ from the center of the knife edge, for phases $\phi=0$ and $\pi / 2$.

Overall, the results are qualitatively similar to those for the near-critical Gaussian experiments. There is, however, some notable disagreement between experi-
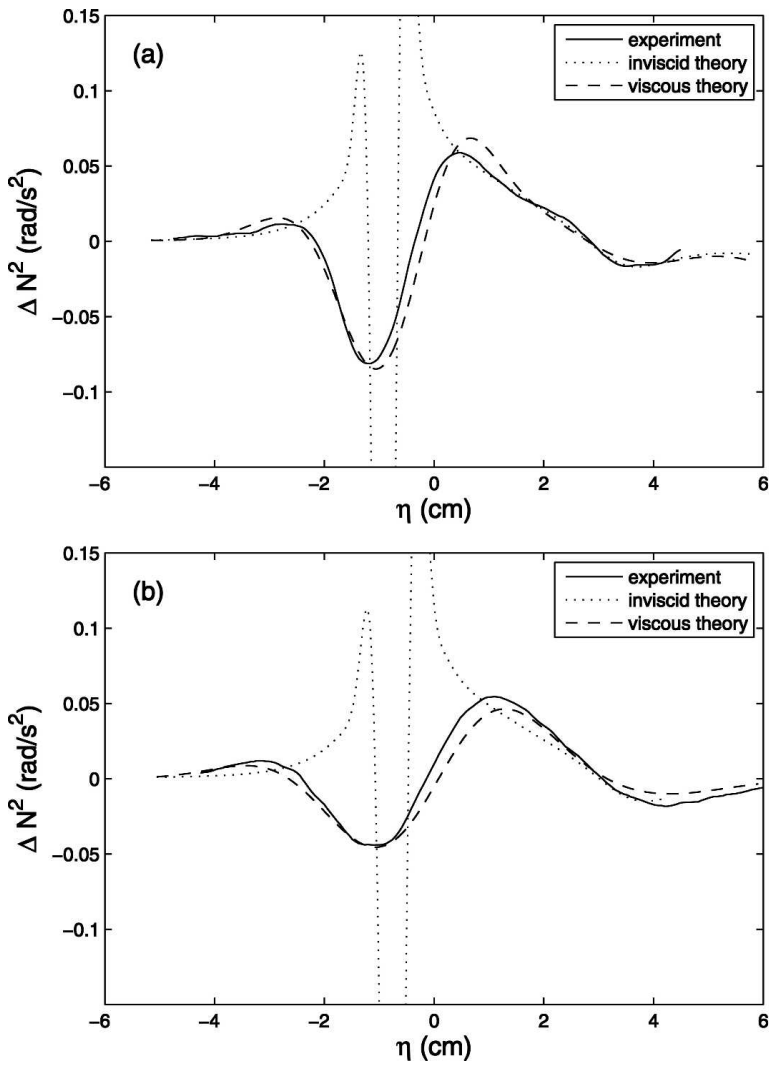

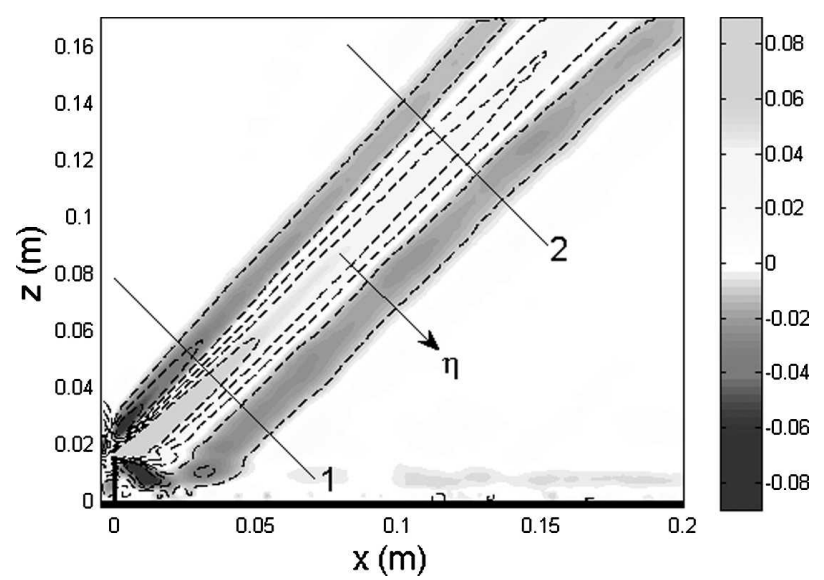

FIG. 6. Experimental $\Delta N^{2}$ field for the knife edge, with $\phi=0$ and $\theta=44.9^{\circ}$. Lines 1 and 2 indicate the location of crosssectional profiles. Contours are plotted at intervals of 0.02 between -0.05 and 0.05 .

ment and theory along the length of the lower half of the beam $(\eta>0)$; experimental values of $\Delta N^{2}$ are lower than theoretical predictions and the experimental beam is somewhat broader. We recognize that this part of the beam comprises both waves that travel directly upward from the knife edge and waves that propagate down
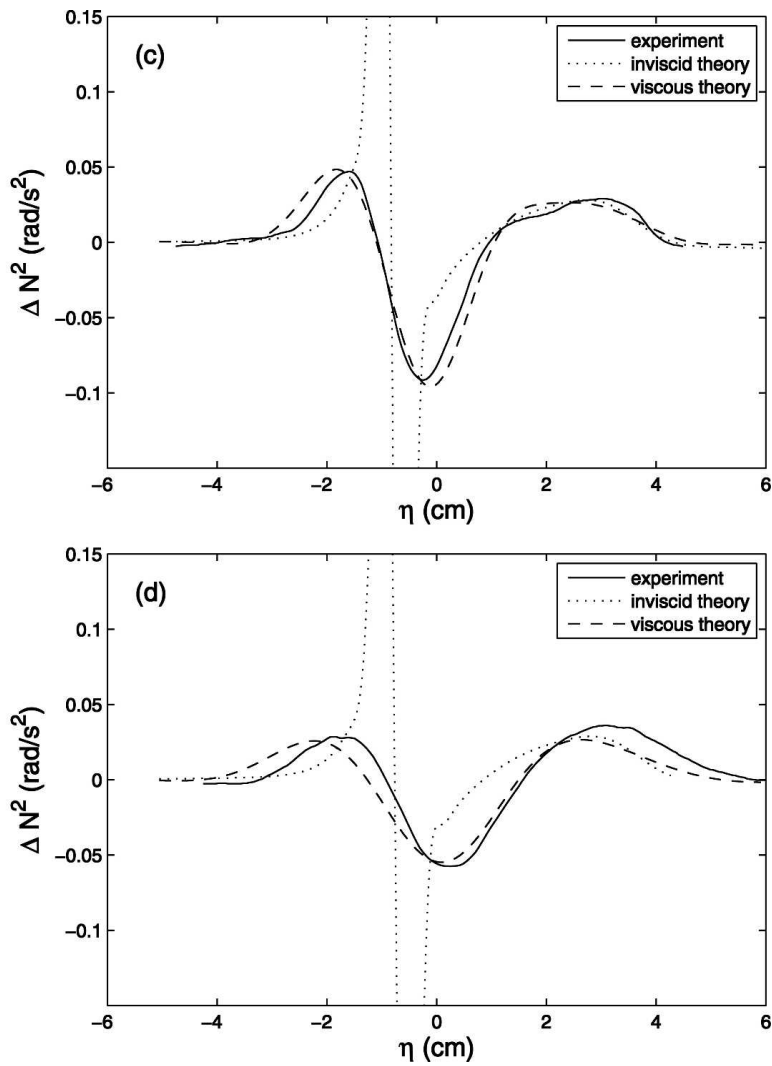

FIG. 5. As in Fig. 3, but for cross sections 1 and 2 in Fig. 4. 

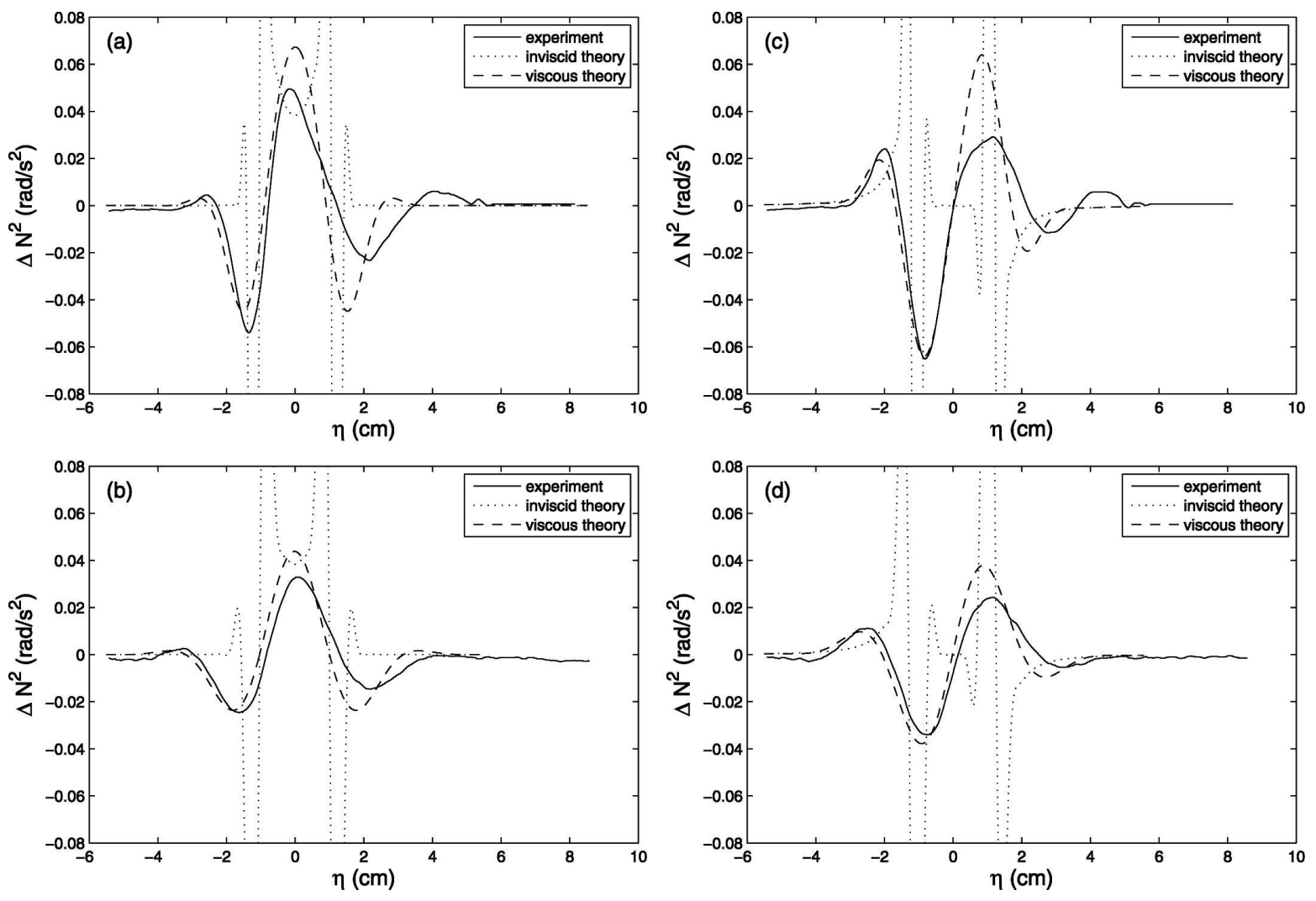

FIG. 7. As in Fig. 3, but for cross sections 1 and 2 in Fig. 6.

from the knife edge and reflect back up from the topographic floor. The viscous correction that we have applied does not take account of the different pathlengths, and we attribute the discrepancy to this shortcoming of the theoretical method. Our hypothesis is supported by the agreement between experiment and theory in the upper half of the beam, which comprises only waves that propagate directly upward from the knife edge.

\section{Conclusions}

We have presented the first set of quantitative experimental results for the generation of internal wave beams by two-dimensional Gaussian and knife-edge topographic features. The synthetic Schlieren technique was used to obtain cross-sectional profiles of perturbations to the stratification $\Delta N^{2}$ in the wave beams generated by Gaussian and knife-edge topography, and the data was used to test two recent, linear models of tidal conversion. By accounting for the effect of viscosity, the experimental results were found to be in agreement with theory, therefore providing support for the use of linearized analytical models to estimate tidal conversion rates by bathymetry.
Without any viscous smoothing, supercritical obstacles like the knife edge scatter wave beams that create singular buoyancy gradients. In the present experiments, viscous smoothing heals those singularities. At the same time, it also appears to remove the secondary instabilities to which inviscid wave beams are susceptible (Balmforth et al. 2002; Petrelis et al. 2006) and that may play an important role in driving oceanic mixing (Lueck and Mudge 1997; Lien and Gregg 2001; Rudnick et al. 2003). We are currently working on extensions of the experiments in which we aim to generate and study these instabilities.

Acknowledgments. This work was supported by ONR Grant N00014-05-1-0575.

\section{REFERENCES}

Baines, P. G., 1982: On internal tide generation models. Deep-Sea Res., 29, 307-338.

Balmforth, N. J., G. R. Ierley, and W. R. Young, 2002: Tidal conversion by nearly critical topography. J. Phys. Oceanogr., 32, 2900-2914.

Dalziel, S. B., G. O. Hughes, and B. R. Sutherland, 2000: Whole field density measurements by synthetic schlieren. Exp. Flu$i d s, \mathbf{2 8}, 322-335$.

Garrett, C., and E. Kunze, 2007: Internal tide generation in the deep ocean. Ann. Rev. Fluid Mech., 29, 57-87. 
Gostiaux, L., and T. Dauxois, 2007: Laboratory experiments on the generation of internal tidal beams over steep slopes. Phys. Fluids, 19, 028102, doi:10.1063/1.2472511.

Holloway, P. E., and M. A. Merrifield, 1999: Internal tide generation by seamounts, ridges and islands. J. Geophys. Res., 104, $25937-25951$.

Hurley, D. G., and G. Keady, 1997: The generation of internal waves by vibrating elliptic cylinders. Part 2: Approximate viscous solution. J. Fluid Mech., 351, 119-138.

Lien, R. C., and M. C. Gregg, 2001: Observations of turbulence in a tidal beam and across a coastal ridge. J. Geophys. Res., 106, 4575-4591.

Llewellyn Smith, S. G., and W. R. Young, 2003: Tidal conversion at a very steep ridge. J. Fluid Mech., 495, 175-191.

Lueck, R. G., and T. D. Mudge, 1997: Topographically induced mixing around a shallow seamount. Science, 276, 1831-1833.

Nash, J. D., E. Kunze, C. M. Lee, and T. B. Sanford, 2006: Structure of the baroclinic tide generated at Keana Ridge, Hawaii. J. Phys. Oceanogr., 36, 1123-1135.
Oster, G., 1965: Density gradients. Sci. Amer., 213, 70-76.

Petrelis, F., S. G. Llewellyn Smith, and W. R. Young, 2006: Tidal conversion at a submarine ridge. J. Phys. Oceanogr., 36, 1053-1071.

Rudnick, D. L., and Coauthors, 2003: From tides to mixing along the Hawaiian Ridge. Science, 301, 355-357.

St. Laurent, L., S. Stringer, C. Garrett, and D. Perrault-Joncas, 2003: The generation of internal tides at abrupt topography. Deep-Sea Res., 50, 987-1003.

Sutherland, B. R., and P. F. Linden, 2002: Internal wave excitation by a vertically oscillating elliptical cylinder. Phys. Fluids, 14, 721-731.

—, S. B. Dalziel, G. O. Hughes, and P. F. Linden, 1999: Visualization and measurement of internal waves by "synthetic schlieren." Part 1: Vertically oscillating cylinder. J. Fluid Mech., 390, 93-126.

Zhang, H. P., B. King, and H. L. Swinney, 2007: Experimental study of internal gravity waves generated by supercritical topography. Phys. Fluids, 096602, doi:10.1063/1.2766741. 